\title{
High risk of depression among low-income women raises awareness about treatment options
}

by Amy Block Joy and Mark Hudes

Depression in young women living in poverty has devastating consequences if left untreated. Lowincome women are at a higher risk for depression than other income groups, and the majority of these women are untreated. The Center for Epidemiologic Studies Depression Scale was used to assess depression symptoms in women under 45 years old in five California counties. More than $45 \%$ were identified as depressed, and the highest level of depressive symptoms was found in Sonoma County. We also found a significant negative association between the number of people in the household and depressive symptom scores, that is, with fewer people in the household depression increased. This result may indicate the need for a support system that is more accessible to individuals in smaller households or it may be an artifact of Hispanics having larger families and lower depression scores. Our results indicate that there is a critical need to provide mental health resources to low-income women, especially those with young children.

\section{$\mathrm{D}$} epression among low-income women remains an unacknowledged and devastating illness. The consequences include low self-esteem, acute and chronic illnesses, relationship conflicts, poor health, financial instability, emotional insecurity and unemployment. Depression affects the whole family, yet it is a treatable condition. Among low-income populations, there is a need for awareness, screening programs and treatment services.

Low-income women are at a higher risk for depression than women in other

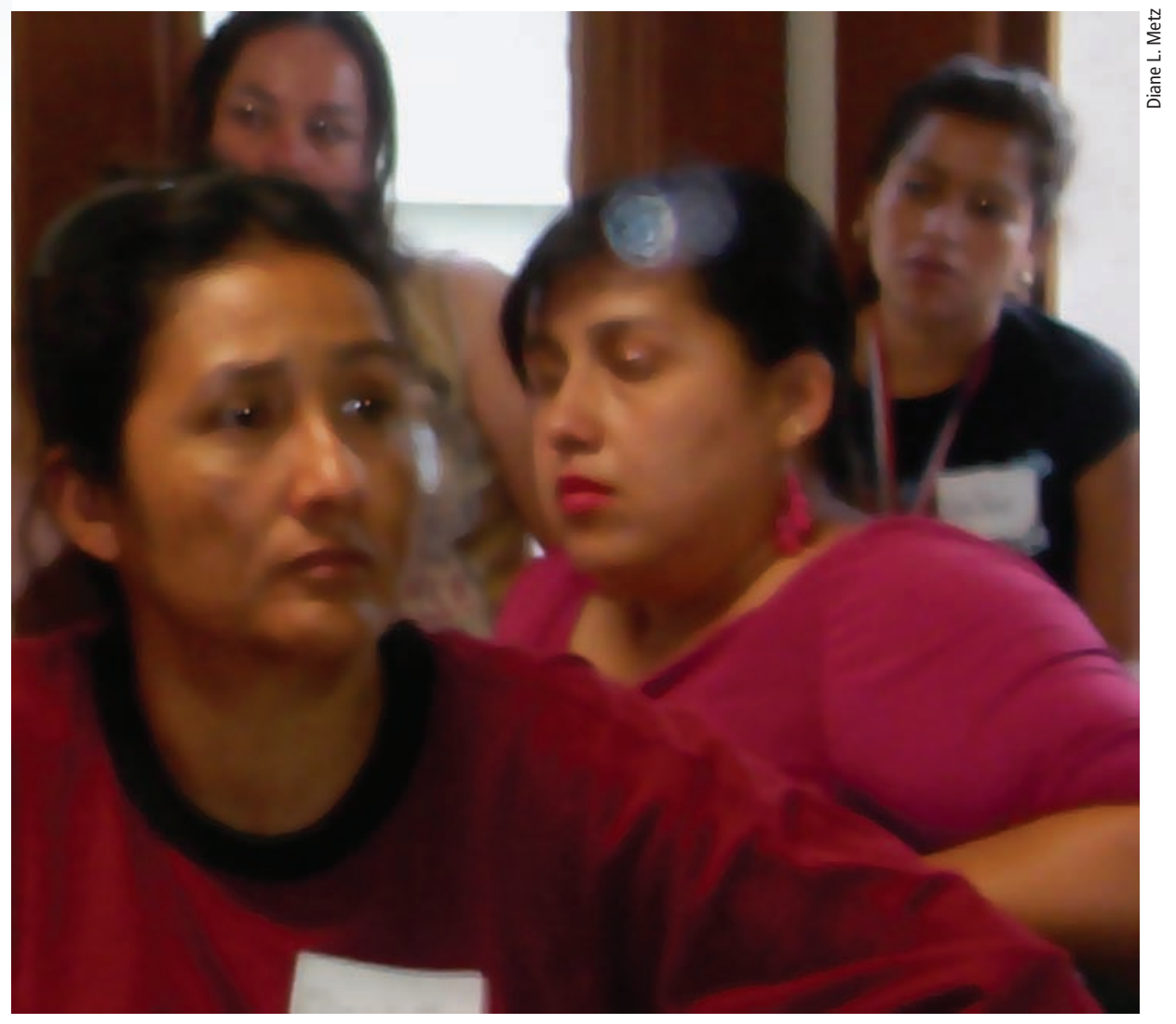

Low-income women have a higher risk for depression than women in other income groups. The consequences of depression include fatigue, poor health and low self-esteem. Depression also increases the risk of financial stress and unemployment.

income groups, and the majority of affected women are untreated (Lennon et al. 2001; Hall 1990; Gadalla 2008). The prevalence of depression in low-income populations has been documented. In one study, the depression rate for women of all income groups $(n=7,000$, ages 15 to 46 years) was $20.4 \%$ (Chen et al. 2005). A report on the prevalence, treatment and consequences of depression highlighted the high rate in low-income women, with a median of $47 \%$ (Lennon et al. 2001). Higher rates of depression have been reported in low-income minority groups, specifically blacks, as well as young mothers and women without a social support network (McBarnette 1996; Chung et al. 2004).

Health care providers who work with low-income families often see a multitude of needs, and food and shelter are usually the first step in treating the effects of poverty. Yet, in battling with daily economic and health con- cerns, women caught in the cycle of poverty can experience acute stress, despair, loss of self-worth and chronic hopelessness.

The disabling effects of depression are numerous and well known. Symptoms include loss of motivation and interest, mood changes and irritability, fatigue, loss of energy and sleep, changes in appetite, poor concentration and forgetfulness, profound sadness, and loss of self-worth and self-esteem. In addition, many people who suffer from depression are also at risk for anxiety symptoms, including fearfulness, worries, headaches, insomnia, fatigue and multiple physical symptoms (e.g., chest pain, palpitations, sweating and alarm).

However, once identified, there are effective treatments for both anxiety and depression. The purpose of this study was to evaluate depression among low-income women in California, and raise awareness of the 
critical need for screening and treatment options.

\section{Assessing depression}

A short, easy-to-administer questionnaire - the Center for Epidemiologic Studies Depression Scale (CES-D) was used to assess depression symptoms in low-income women (Radoff 1977). The National Institute of Mental Health's Center for Epidemiologic Studies developed the self-report instrument to measure the frequency and severity of 20 common depression symptoms. Using a four-point Likert scale, the response for each symptom is scored and the scores (ranging from 0 to $60)$ calculated. Scores of 16 or greater indicate depression. The reliability of the instrument is 0.85 (internal consistency, coefficient alpha, Spearman-Brown).

This instrument has been demonstrated to be reliable and valid for low-income populations (Radoff 1977; Thomas et al. 2001). In a study of 179 low-income women attending primary care clinics (ages 20 to 77), the cutoff score of 16 or higher was validated (sensitivity $=0.95$, specificity $=0.70$ ) for diagnosing major depressive disorder (MDD) in low-income populations (Thomas et al. 2001). Research has shown that the scores of clinically depressed individuals are much higher than those of nondepressed individuals. Furthermore, researchers have validated this instrument in other ethnic groups: $65 \%$ of low-income mothers, mainly blacks, were identified as being depressed in a study using the CES-D screener (Chung et al. 2004).

The self-report instrument has been widely used in both community and population studies to assess depressive symptoms and to identify the proportion of individuals with major depressive disorders. Radoff (1977) examined the structure of the screener (questionnaire). Her four-factor structure -

(1) depressed affect, (2) positive affect, (3) somatic complaints and (4) interpersonal problems - has been replicated and is widely accepted. Another study validated the use of this screener and the four-factor structure for low-income blacks (Nguyen et al. 2004). The general factor structure is also considered adequate for Mexican Americans, blacks and whites

Women caught in the cycle of poverty can experience acute stress, despair, loss of self-worth and chronic hopelessness.

(Roberts 1980) but not for Native

Americans (Chapleski et al. 1997).

\section{Depression screening}

CES-D questionnaires were initially completed by 157 low-income women as part of a nutrition-education study (Cena 2007; Cena et al. 2007; Cena et al. 2008a, 2008b). In all, the researchers studied 14 variables that included demographic factors (geographical location, language spoken, ethnicity, low-income status, household size, age and participation in the federal Special Supplemental Program for Women, Infants and Children [WIC] and Food Stamp Program), folic acid intake (natural-food folic acid, synthetic folic acid [SFA] from fortified foods, synthetic folic acid from supplements, total folic acid from all sources, folate status) and depression. The folic acid variables were added to determine if there was a relationship between folic acid intake and depression. Since folic acid has a role in maintaining serotonin — the major neurotransmitter needed to maintain a feeling of "well-being" — researchers have hypothesized that folic acid may reduce depression. However, studies have found both a positive and inverse relationship between folic acid and depression (Astorg et al. 2008; Coppen and BolanderGouaille 2005; Taylor et al. 2004).

Most food sources of folic acid are considered healthy foods and include breakfast cereals (cold cereals, oatmeal, grits, Cream of Wheat), eggs, beans, green vegetables, tortillas and other breads (slices, rolls, bagels, muffins, buns), oranges and orange juice. Some of these foods - breads, orange juice and breakfast cereals - are fortified with synthetic folic acid.

Demographics. The women in the study $(n=157)$ were low-income clients enrolled in a nutrition-education program for food-stamp-eligible recipients; $52.9 \%$ were enrolled in WIC with incomes less than $185 \%$ of the poverty level, 39.5\% were enrolled in the federal Food Stamp Program with incomes less than $130 \%$ of the poverty level, and $73 \%$ were receiving either or both WIC and
Food Stamp Program benefits.

The sample size per county was Fresno, 39; Sonoma, 41; Shasta/Trinity, 36; and San Diego, 41. The women were 18 to 45 years of age (average $=$ 31.6 years) and had diverse ethnicity (56.7\% Hispanic, 35.7\% non-Hispanic white, 3.8\% Native American/Alaskan Native, $1.3 \%$ Asian/Pacific Islander, 2.5\% mixed ethnicity).

All participants in Fresno and San Diego counties were self-identified as Hispanic and completed the screener in Spanish (51\%), while all the participants in Shasta/Trinity and Sonoma counties identified themselves as a number of ethnicities and completed the screener in English (49\%). All subjects signed a consent form, and the Institutional Review Board of UC Davis approved the study.

Statistical analysis. Of the 157 clients enrolled in the study, 121 completed the CES-D survey. Thirty-six participants were not included in the analysis due to incomplete survey responses or incorrectly marked multiple answers for at least one question on the instrument.

Statistical tests were performed using SPSS version 15.0 (SPSS, Chicago, IL, 2006) to examine relationships between depression and 14 variables. Two-sample independent $t$-tests were employed to evaluate depressive symptoms by each dichotomous variable (language, food stamps, WIC participation). For categorical variables (county, ethnicity, folate intake), one-way analysis-of-variance techniques were used, and, if significant, followed up using Tukey's post-

TABLE 1. Depression scores by county
and language


hoc tests for multiple comparisons. We examined the relationship between depression score and continuous variables (e.g., folate intake, household size) utilizing Pearson correlation coefficients. Statistical significance was defined as $P$ $<0.05$, and results where $0.05<P<0.06$ were considered to be approaching statistical significance.

Referrals. Program staff provided the study participants with referrals to free mental-health resources in their local communities. The researchers hypothesized that the CES-D screener might raise awareness in study participants, and this awareness might lead to increased stress. Staff members were trained to listen for questions about depression, and a referral handout was given to participants after they completed the screener. Due to confidentiality, the participants were not asked about their use of referrals, and staff did not identify any mental health issues related directly to depression.

\section{High depression scores}

Of the 121 participants who completed the CES-D screener correctly, scores ranged from 0 to 44 with a mean of 16.1 (standard deviation of 10.4). As described in the literature, CES-D scores of 16 or more were classified as "depressed" and scores below 16 were "not depressed," so that $45.5 \%$ of the study participants were identified as depressed (Cena et al. 2007).

Language. The results demonstrated some associations between depressive symptoms and the respondent's language (table 1). Of all the sites studied, Sonoma County $(n=41)$ had the highest level of depressive symptoms, and all were English-speaking women. San Diego County $(n=41)$ scores were lower, and all were Spanish speakers

$\begin{aligned} & \text { TABLE 2. Statistical associations between folate } \\ & \text { foods and household size and depression } \\ & \text { measured by CES-D scores } \\ & \text { in low-income women }(n=121)\end{aligned}$
$\begin{aligned} & \text { Variable } \\ & \text { Correlation with } \\ & \text { CES-D score }(r) \quad P \text { value* }\end{aligned}$
$\begin{aligned} & \text { Folate from } \\ & \text { fortified foods (SFA) }\end{aligned}$
$\begin{aligned} & \text { Number of people } \\ & \text { in household }\end{aligned}$
$\begin{aligned} & \text { * Statistical significance was defined to be } P<0.005 \\ & P \text { is the likelihood of observing the result by chance alone. }\end{aligned}$

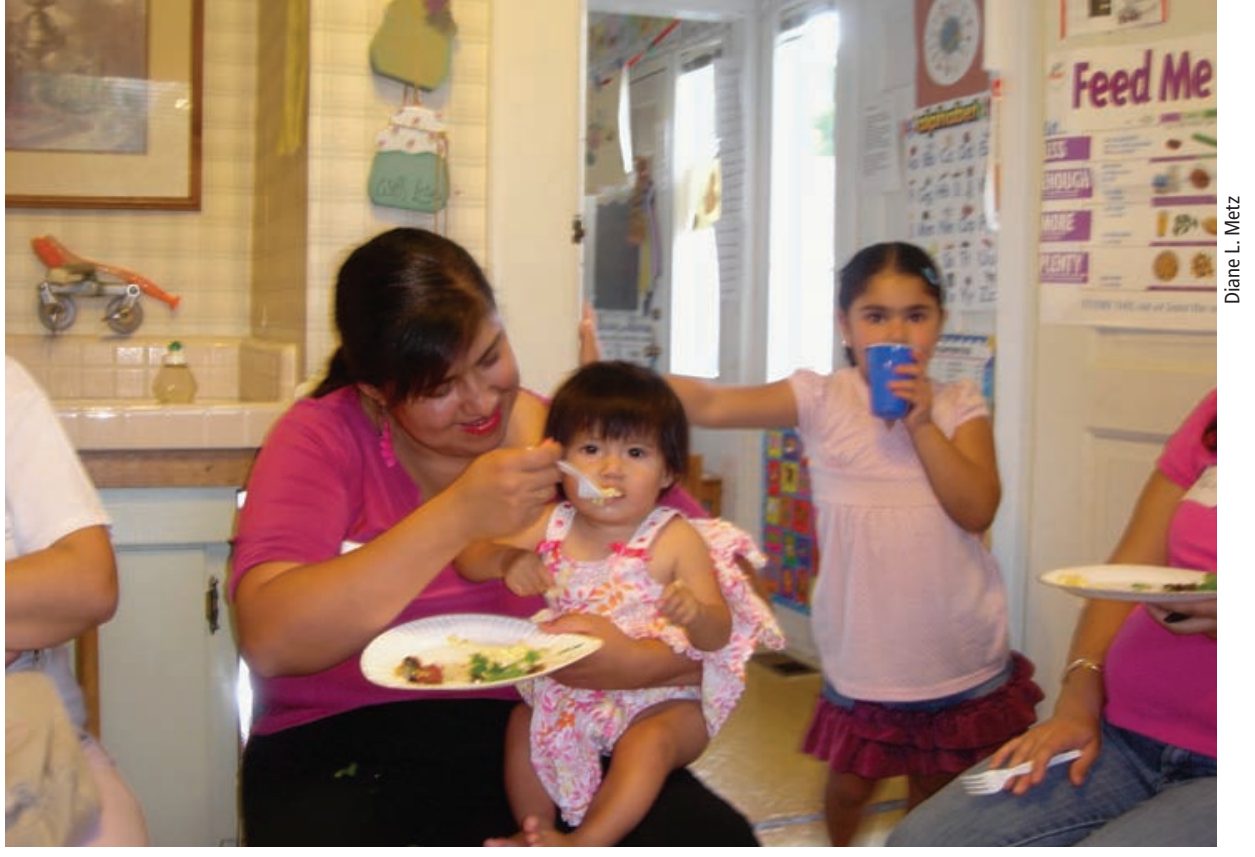

Depression is a treatable illness; free counseling services are available through community referrals in every California county.

(table 1). There was a significant difference in the depressive symptom scores between Sonoma and San Diego counties $(P=0.018)$ and a near-significant difference between English and Spanish language $(P=0.059)$.

Household size. The average household size was 4.3 people $( \pm 1.7)$, with means of $4.9( \pm 1.5)$ people for Hispanic women and $3.3( \pm 1.6)$ for non-Hispanic white women. Household size was significantly different among Hispanic clients compared to non-Hispanic white clients $(P<0.001)$. In addition, there was a negative association between the number of people in the household and depressive symptoms that trended toward statistical significance $(P=0.056)$ (table 2$)$. A possible explanation is that more than half the women were Hispanic, with larger household sizes and lower CES-D scores.

Folic acid intake. When the women were divided into two groups (depressed and nondepressed) and compared for folic acid intake, there were no significant differences between groups. This is consistent with other published studies (Astorg et al. 2008). In a longitudinal study of young women $(n=5,051$, ages 20 to 34 years), low blood folic acid levels were not significantly associated with depression (Kendrick et al. 2008). In a study of pregnant women, depression was not associated with folic acid intake ( $n=1,277$ women, with $8.1 \%$ classified as depressed) (Cho et al. 2008).

However, we found a significant positive association between depression and foods fortified with synthetic folic acid when CES-D score was treated as a continuous variable $(r=0.277, P=$ 0.002) (table 2). Women who reported more synthetic folic acid intake also had higher depression scores. Previous research documents a positive as well as an inverse association between depression and folic acid.

Folic acid is recommended for women of childbearing age to prevent birth defects. The relationship between folic acid and depression is still under debate. Three published studies have found an inverse association between blood levels of folate and depression, that is, depressed individuals have been shown to have low blood folate levels (Coppen and Bolander-Gouaille 2005; Taylor et al. 2004; Morris et al. 2003). Some doctors recommend folic acid supplementation to enhance the treatment of depression by improving the effectiveness of some antidepressant medications (Coppen and BolanderGouaille 2005; Taylor et al. 2004).

\section{Depression trends}

The National Center for Children in Poverty used the CES-D instrument to study the prevalence of depression in low-income U.S. women, and found that $12.9 \%$ had experienced major depressive disorder for the past 12 months (Lennon et al. 2001). For women in this study the percentage was even higher: in the five counties that we studied, $45.5 \%$ were identified as depressed using the criteria defined in the CES-D screener.

Of the 14 variables studied, only two showed significant trends. There was a significant negative association between 
the number of people in the household and depressive symptom scores; that is, with fewer people in the household, depression increased. This result may indicate the need for a support system that is more accessible to individuals in smaller households. Researchers have documented that Hispanic women with social-support networks, such as those provided by larger families, have reduced depression risk compared to other ethnic groups (Samaan 2000), suggesting that they have better coping strategies due to a variety of factors including social, religious and spiritual roots and extended family support. In this study, the household size of 4.9 persons for Hispanic clients was significantly larger than the 3.3-person households of nonHispanic white clients $(P<0.001)$.

\section{Treating low-income women}

Numerous treatment options are available for depression, and successful results in low-income populations have been published. The main options are counseling (individual and group) and medication (a number of antidepressants and antianxiety medications have been tested). In a study on the treatment preferences of 1,893 low-income women, 78\% selected counseling, 55\% chose group counseling and $32 \%$ chose medication (Nadeem et al. 2008). In a sample of 267 low-income minority women who were randomly assigned to antidepressant medications, psychotherapy or community referrals, researchers found that both the medication and therapy treatments significantly reduced depressive symptoms. After a year of treatment, $50.9 \%$ of the medication group and $56.9 \%$ of the therapy group were no longer clinically depressed. Depression in the community-referral group (control) was not significant (Miranda et al. 2006). Other research has shown similar results in low-income black and Latino populations (Green et al. 2006).

Our results indicate that there is an important need to provide information on mental health programs to low-income women. Further work is needed to determine the feasibility of treating depression by identifying the need through screening programs and providing referrals. Depression is a treatable condition, and linking women and others to free treatment services is essential. In addition, information about depression, its symptoms and treatment options should be provided in public health and antipoverty programs, including for women in low-income agricultural jobs and communities.

A. Block Joy is Cooperative Extension Specialist in Nutritional Ecology, UC Davis; and M. Hudes is Senior Statistician, Department of Nutritional Sciences and Toxicology, UC Berkeley.

Funding for this study was provided in part by the USDA Fellowship in Human Nutrition (grant number 02-38420-11727), USDA/FSNE and the Department of Nutrition, UC Davis. Emily Cena analyzed the data in consultation with the authors as part of her doctoral thesis in Nutritional Biology at UC Davis. We also acknowledge county personnel who contributed to field implementation and data collection: Fresno (Connie Schneider, Patty Davidson, Yolanda Lopez), Shasta/Trinity (Gloria Espinosa Hall, Lori Coker, Tamila Medinnus), San Diego (Patti Wooten Swanson, Lydia Lopez, Lori Renstrom, Margarita Schwarz) and Sonoma (Linda Garcia, Wanda Tapia, Carla Vaughn).

\section{References}

Astorg G, Couthouis A, deCourcy GP, et al. 2008 Association of folate intake with the occurrence of depressive episodes in middle-aged French men and women. Brit J Nutr 100(1):183-7.

Cena ER. 2007. Folate intake, food-related behaviors, and nutrition education in low-income women of childbearing age. Ph.D. dissertation, Department of Nutritional Biology, Office of Graduate Studies, UC Davis.

Cena ER, Joy AB, Heneman K, et al. 2008a. Folate intake and food-related behaviors in nonpregnant, low-income women of childbearing age. J Am Diet Assoc 108:1364-8.

Cena ER, Joy AB, Heneman K, et al. 2008b. Learnercentered nutrition education improves folate intake and food-related behaviors in nonpregnant, lowincome women of childbearing age. J Am Diet Assoc 108:1627-35

Cena ER, Joy AB, Heneman K, Zidenberg-Cherr S. 2007. Low-income women in California may be at risk of inadequate folate intake. Cal Ag 61:85-9.

Chapleski EE, Lamphere JK, Kaczynski R, et al. 1997. Structure of a depression measure among American Indian elders: Confirmatory factor analysis of the CES D Scale. Res Aging 19:462-85.

Chen YY, Subramanian SV, Acevedo-Garcia D, Kawach I. 2005. Women's status and depressive symptoms: A multilevel analysis. Soc Sci Med 60(1):49-60

Cho YJ, Han Jy, Cho JS, et al. 2008. Prenatal multivitamins containing folic acid do not decrease prevalence of depression among pregnant women. J Obstet Gynaecol 28(5):482-4.
Chung EK, McCollum KF, Elo IT, et al. 2004. Maternal depressive symptoms and infant health practices among low-income women. Pediatrics 113(6): e523-9.

Coppen A, Bolander-Gouaille C. 2005. Treatment of depression: Time to consider folic acid and vitamin B-12. J Psychopharmacol 19(1):59-65.

Gadalla TM. 2008. Comparison of users and non-users of mental health services among depressed women: A national study. Women Health 47:1-19.

Green BL, Krupnick JL, Chung J, et al. 2006. Impact of PTSD comorbidity on one-year outcomes in a depression trail. J Clin Psychol 62(7):815-35.

Hall LA. 1990. Prevalence and correlations of depression symptoms in mothers of young children. Public Health Nurs 7(2):71-9.

Kendrick T, Dunn N, Robinson S, et al. 2008. A longitudinal study of blood folate levels and depression symptoms among young women in the Southampton Women's Survey. J Epidemiol Commun Health 62(11):966-72.

Lennon MC, Blome J, English K. 2001. Depression and low-income women: Challenges for TANF and Welfareto-Work policies and programs. Research Forum on Children, Families and New Federalism; National Center for Children in Poverty. Columbia University Report.

McBarnette LS. 1996. African American women. In: Bayne-Smith M (ed.). Race, Gender, and Health. Newbury Park, CA: Sage Pub. p 43-67.

Miranda J, Green BL, Krupnick JL, et al. 2006. One-yea outcomes of a randomized clinical trial treating depres- sion in low-income minority women. J Consult Clin Psychol 74(1):99-111.

Morris MS, Fava M, Jacques PF, et al. 2003. Depressed folate status in the U.S. population. Psychother Psychosom 72(2):80-7.

Nadeem E, Lange JM, Miranda J. 2008. Mental health care preferences among low-income and minority women. Arch Womens Ment Health 11(2):93-102

Nguyen HT, Kitner-Triolog M, Evans MK, et al. 2004 Factorial invariance of the CES-D in low socioeconomic status African Americans compared with a nationally representative sample. Psychiat Res 126:177-87.

Radoff LS. 1977. The CES-D scale: A self-report depression scale for research in the general population. Appl Psych Meas 1(3):385-401.

Roberts RE. 1980. Reliability of the CES-D scale in different ethnic contexts. Psychiat Res 2(2):125-34.

Samaan RA. 2000. The influence of race, ethnicity, and poverty on the mental health of children. Health Care Underserved 11:100-10.

Taylor MJ, Carney SM, Goodwin GM, et al. 2004 Folate for depressive disorders: Systematic review and meta-analysis of randomized controlled trials. J Psychopharmacol 18(2):251-6.

Thomas JL, Jones GN, Scarinci IC, et al. 2001. The utility of the CES-D as a depression screening measure among low-income women attending primary care clinics. Int J Psychiat Med 31(1):25-40. 